\title{
Diagnosing posttraumatic stress disorder in refugees
}

Shafaz Veettil, Anastasiya Vinokurtseva

\begin{abstract}
Global crises has amounted to the forced international displacement of $\mathbf{2 5 . 4}$ million refugees. Refugees from conflictaffected areas are especially vulnerable to posttraumatic stress disorder (PTSD) compared to the general population due to their past and present hardships and history of trauma. PTSD is characterized by a constellation of symptoms identified by the Diagnostic and Statistical Manual of Mental Disorders (DSM). DSM-5 departed from DSM-IV by reclassifying PTSD as a trauma- and stressor-related disorder and introducing a fourth symptom cluster-negative alterations in mood/cognitionto the previous three-symptom cluster model. In severely traumatized refugees, this new cluster exhibited relatively high sensitivity, specificity, positive predictive power, and negative predictive power-in concordance with the range of symptoms exhibited by this population-and allowed for the applicability of the DSM-5 criteria. However, the Western sample basis of the DSM-5 might make it inferior to alternative models as a diagnostic tool for PTSD in refugees and as a springboard for treatment. In addition (and possibly due) to PTSD, refugees are at high risk for mental health distress and suffer from poor health outcomes. Optimizing diagnostic criteria and overcoming barriers to diagnosis and access to care would benefit patients and facilitate treatment.
\end{abstract}

\section{INTRODUCTION}

The world is currently facing a humanitarian crisis, to a degree unprecedented since the Second World War., ${ }^{1,2}$ Troubled by persecution, war, and violence, refugees have felt the widereaching impact of this crisis and suffered forced displacement from their home countries as a result. According to the United Nations High Commissioner for Refugees, there are 25.4 million refugees worldwide and over 100000 now living in Canada. ${ }^{3}$ Many of these refugees received by Canada in recent years came from Syria, Eritrea, Iraq, and Afghanistan. ${ }^{4}$ This paper will introduce psychosocial triggers and posttraumatic stress disorder (PTSD) in relation to refugees, cover the diagnosis of PTSD, and discuss further considerations that need to be taken into account during the provision of care. A potential future in which biomarkers are used to diagnose PTSD in refugees will also be touched on briefly.

\section{PSYCHOSOCIAL STRESSORS AND PTSD}

Refugees fall under the umbrella term newcomer, which covers additional groups including immigrants and asylum seekers. ${ }^{5}$ Even when compared to other newcomers, refugees present unique and complex challenges to clinicians, particularly for those unaccustomed to serving them. ${ }^{6}$ The distinct refugee journey-from premigration to resettlement and beyond-exposes individuals to multiple stressors and indelible trauma..$^{7-11}$ Examples of stressors include violence, harmful living conditions, poverty, instability, separation and loss, isolation, and acculturation. While such exposures may not necessarily lead to PTSD, stressors and traumatic episodes are required for the condition to occur. ${ }^{12,13}$

Greater exposure to premigration traumatic experiences and postmigration stress are consistent predictors for the development of PTSD in refugees. ${ }^{9}, 12$ Accordingly, there is a higher prevalence rate of PTSD in refugees than in other groups-even across different stages, cultures, and contexts-and elevated rates of other psychological disorders. ${ }^{12-14}$ In samples of various different refugee populations, prevalence rates ranged from $4 \%$ to $86 \%$, though a systematic review suggested that methodological inconsistencies were partially responsible for these heterogenous figures. ${ }^{12}$

\section{DIAGNOSING PTSD}

In 2013, the fifth edition release of the Diagnostic and Statistical Manual of Mental Disorders (DSM-5) brought along with it a change in the diagnostic criteria for PTSD. ${ }^{11}$ DSM-5 reclassified PTSD from an anxiety disorder to a trauma- and stressor-related disorder, added and removed certain individual symptoms, and replaced the previous three-symptom cluster model (reexperiencing, avoidance, and hyperarousal) with a four-symptom cluster model (reexperiencing, avoidance, negative alterations in mood/cognition, and hyperarousal). ${ }^{11}$

While previous studies found comparable rates of PTSD between DSM-IV and DSM-5 criteria in non-refugees, fewer participants of a Swiss-based study involving severely traumatized refugees met the DSM-5 diagnostic criteria for PTSD versus the DSM-IV criteria. ${ }^{11}$ This result was surprising because DSM-5's inclusion of the "negative alterations in mood/cognition" cluster was expected by the study authors to be pertinent to refugeesas the prolonged and repeated trauma relevant to individuals of refugee backgrounds can manifest as anger, guilt, and emotional dysregulation-and contribute to more probable cases of PTSD. ${ }^{15}$

In reality, the "negative alterations in mood/cognition" cluster did indeed demonstrate high sensitivity, specificity, positive predictive power, and negative predictive power. ${ }^{11}$ The discrepancy in diagnostic rates between the DSM-IV and DSM-5 models were instead likely due to changes to symptom descriptions and the exclusion of the symptom of "a sense of a foreshortened future." The authors of the study ultimately determined that the DSM-5 symptom structure remained a good fit for traumatized refugees.

Interestingly, an Australian-based study revealed that while the DSM-5 criteria was adequate for this population, it was the poorest fit compared to four alternative models. ${ }^{14}$ Instead, the 
authors identified the competing six-symptom cluster Anhedonia model (reexperiencing, avoidance, negative affect, anhedonia, dysphoric arousal, and anxious arousal) as the superior model for diagnosing PTSD in refugees. ${ }^{14}$ This result was consistent with an earlier study looking at Burmese refugees in Thailand that found an approximation of the DSM-5 criteria as adequate but inferior to competing models. ${ }^{16}$ The implication of these two studies is that the Western sample based DSM-5 criteria may not optimally reflect the symptom clusters expressed in refugees suffering from PTSD that come from non-Western countries. Another implication is that refugees may be better served during treatment of PTSD symptoms when using these alternative models.

\section{FURTHER CONSIDERATIONS}

PTSD does not exist in isolation in refugees., ${ }^{5,1-13,15}$ It often coincides with mood disorders, anxiety disorders, and depression and may contribute to increased mental health distress in refugee post-partum women and refugee youth. Post-partum refugee women in Canada are more likely to experience depression, have a psychiatric emergency department visit, and have a psychiatric hospitalization than their immigrant and non-refugee counterparts. ${ }^{17-19}$ Refugee youth, meanwhile, rely more on the emergency department as a first mental health contact than immigrant and non-refugee youth. ${ }^{20}$ Although the exact role of trauma in health status is unclear, other health issues documented in refugees are increased morbidity, decreased life expectancy, and elevated acute and chronic health disparities., ${ }^{2,1321-23}$

Poor health outcomes, comorbidities, and greater reliance on the emergency department highlight the importance of properly diagnosing PTSD in this population and the need to address barriers in care. However, competing classification systems and models for PTSD define trauma and conceptualize symptoms differently, as discussed in greater detail earlier, which can lead to underdiagnosis and has consequences on treatment. ${ }^{13}$ Diagnostic discordance in up to $30 \%$ of patients occurs between DSM-IV and DSM-5 PTSD criteria, illustrating the magnitude of effect changes in diagnostic formulation can have. ${ }^{13}$ The misclassification rate is similar in young children. In such misdiagnosed children, trauma-related symptoms can go untreated and be perceived as manifestations of other diagnoses. ${ }^{24}$ Complaints about the inadequacy of the DSM-IV PTSD criteria for this age group led to the introduction of PTSD criteria specific for preschool-aged children in DSM-5. ${ }^{25}$ Considering $50 \%$ of refugees are children, this may affect how PTSD in young refugees is studied and diagnosed in the future. ${ }^{26}$

Additionally, having a proper characterization of symptoms is important for treatment itself as current trauma-focused interventions are symptom-oriented..$^{13,15,27}$ Such interventions include Cognitive Behavioral Therapy, Narrative Exposure Therapy, and Eye Movement Desensitization and Reprocessing. These interventions attempt to address the symptoms associated with PTSD by processing traumatic memories and altering maladaptive cognitive behaviors into functional and adaptive ones. While some of these interventions already demonstrate significant promise, a more definitive understanding of PTSD symptoms in refugees would provide clearer targets for clinical intervention.
Symptom disclosure difficulties, possible issues in crosscultural diagnostic validity, normalization of distress, and overt focus on co-occurring somatic complaints can add to the challenges involved in diagnosing PTSD. ${ }^{13}$ Barriers that prevent refugee PTSD patients from accessing care in the first place and contribute to the other poor health outcomes mentioned above include language gaps, discrimination, cultural influence, difficulty navigating the healthcare system, and isolation and lack of social support. ${ }^{5,28}$

\section{FUTURE POSSIBILITIES}

One day, a wide array of biomarkers based on biological disruptions associated with PTSD may supplant DSM criteria as the gold standard for PTSD diagnosis and help overcome some of the diagnostic barriers discussed..$^{13,29,30}$ Having an objective confirmation of PTSD, rather than relying purely on subjective reports from patients, may also help clinicians distinguish PTSD from comorbidities that share overlapping symptoms, identify novel subtypes of PTSD unique to refugees, and inform therapeutic targets. While knowledge of several PTSD-related autonomic and neuroanatomic abnormalities exists, much work is needed to attain a constellation of biomarkers with the sensitivity, specificity, and practicality necessary to clinically diagnose as heterogenous a disorder as PTSD. As the search for biomarkers of PTSD continues, any new insight gathered into how the disorder manifests clinically in refugees would benefit those looking to optimize DSM criteria for this population. A stronger DSM would then better serve patients who lack access to testing for these biomarkers.

\section{CONCLUSION}

Refugees total over 25 million worldwide and are at high risk for PTSD due to previous traumatic events and stressors associated with the protracted journey to resettlement. The DSM-5 criteria represents an adequate clinical lodestar for diagnosing PTSD yet may be inferior to other models due to sample bias. Criteria discrepancies and other factors further complicate diagnosis. Optimizing diagnostic criteria and overcoming diagnostic and healthcare access barriers may promote the mental and general wellbeing of refugee patients.

\section{REFERENCES}

1. Esses VM, Hamilton LK, Gaucher D. The global refugee crisis: empirical evidence and policy implications for improving public attitudes and facilitating refugee resettlement. Soc Issues Policy Rev. 2017 Jan 1;11(1):78-123. https://doi.org/10.1111/sipr.12028

2. Kaur G. Chronic pain in refugee torture survivors. J Glob Health. 2017 Dec;7(2):010303.

3. Mid-Year Trends, June 2017 [Internet]. Geneva (Switzerland): United Nations High Commissioner for Refugees; 2018 Mar [cited 2018 Nov 12]. Available from: https://www.unhcr.org/uk/statistics/ unhcrstats/5aaa4fd27/mid-year-trends-june-2017.html

4. Pottie K, Gruner D, Magwood O. Canada's response to refugees at the primary health care level. Public Heal Res Pract. 2018;28(1). https:// doi.org/10.17061/phrp2811803

5. Kirmayer LJ, Narasiah L, Munoz M, et al. Common mental health problems in immigrants and refugees: general approach in primary care. CMAJ. 2011 Sep 6;183(12):E959-67. https://doi.org/10.1503/ 
cmaj.090292

6. Martin D, Miller AP, Quesnel-Vallée A, et al. Canada's universal healthcare system: achieving its potential. Lancet. 2018 Apr;391(10131):171835. https://doi.org/10.1016/S0140-6736(18)30181-8

7. Perera S, Gavian M, Frazier P, et al. A longitudinal study of demographic factors associated with stressors and symptoms in African refugees. Am J Orthopsychiatry. 2013 Oct 1;83(4):472-82. https://doi.org/10.1111/ajop.12047

8. Alfadhli K, Drury J. A typology of secondary stressors among refugees of conflict in the Middle East: the case of Syrian refugees in Jordan. PLoS Curr. 2018 May;10. https://doi.org/10.1371/currents. dis.4bd3e6437bff $47 \mathrm{~b} 33 \mathrm{dddb} 9 \mathrm{f} 73 \mathrm{cb} 72 \mathrm{f} 3 \mathrm{~d} 8$

9. Chen W, Hall BJ, Ling L, et al. Pre-migration and post-migration factors associated with mental health in humanitarian migrants in Australia and the moderation effect of post-migration stressors: findings from the first wave data of the BNLA cohort study. Lancet Psychiatry. 2017 Mar;4(3):218-29. https://doi.org/10.1016/S22150366(17)30032-9

10. Hollifield M, Warner TD, Lian N, et al. Measuring trauma and health status in refugees. JAMA. 2002 Aug;288(5):611-21. https://doi. org/10.1001/jama.288.5.611

11. Schnyder U, Müller J, Morina N, et al. A comparison of DSM-5 and DSM-IV diagnostic criteria for posttraumatic stress disorder in traumatized refugees. J Trauma Stress. 2015 Aug;28(4):267-74. https:// doi.org/10.1002/jts.22023

12. Bogic M, Njoku A, Priebe S. Long-term mental health of war-refugees: a systematic literature review. BMC Int Health Hum Rights. 2015 Oct;15(1):29. https://doi.org/10.1186/s12914-015-0064-9

13. Yehuda R, Hoge CW, McFarlane AC, et al. Post-traumatic stress disorder. Nat Rev Dis Primers. 2015 Oct;1:15057. https://doi. org/10.1038/nrdp.2015.57

14. Specker P, Liddell BJ, Byrow Y, et al. A factor analytic investigation of DSM-5 PTSD symptoms in a culturally diverse sample of refugees resettled in Australia. Confl Health. 2018 Dec;12(1):26. https://doi. org/10.1186/s13031-018-0155-Z

15. Nickerson A, Bryant RA, Silove D, et al. A critical review of psychological treatments of posttraumatic stress disorder in refugees. Clin Psychol Rev. 2011 Apr;31(3):399-417. https://doi.org/10.1016/j. cpr.2010.10.004

16. Michalopoulos LM, Unick GJ, Haroz EE, et al. Exploring the fit of Western PTSD models across three non-Western low- and middleincome countries. Traumatology. 2015;21(2):55-63. https://doi. org/10.1037/trm0000020

17. Vigod SN, Bagadia AJ, Hussain-Shamsy N, et al. Postpartum mental health of immigrant mothers by region of origin, time since immigration, and refugee status: a population-based study. Arch Womens Ment Health. 2017 Jun;20(3):439-47. https://doi.org/10.1007/ s00737-017-0721-1

18. Khanlou N, Haque N, Skinner A, et al. Scoping review on maternal health among immigrant and refugee women in Canada: prenatal, intrapartum, and postnatal care. J Pregnancy. 2017 Jan;2017:1-14. https://doi.org/10.1155/2017/8783294

19. Stewart DE, Gagnon A, Saucier J-F, et al. Postpartum depression symptoms in newcomers. Can J Psychiatry. 2008 Feb;53(2):121-4. https://doi.org/10.1177/070674370805300208

20. Saunders NR, Gill PJ, Holder L, et al. Use of the emergency department as a first point of contact for mental health care by immigrant youth in Canada: a population-based study. CMAJ. 2018 Oct;190(40):E1183-91. https://doi.org/10.1503/cmaj.180277

21. Cutler SJ. Refugee crisis and re-emergence of forgotten infections in Europe. Clin Microbiol Infect. 2016 Jan;22(1):8-9. https://doi. org/10.1016/j.cmi.2015.10.018

22. Pfeil J, Hufnagel M. Rational diagnostics and therapies in child refugees. Pediatr Infect Dis J. 2018 Mar;37(3):272-4. https://doi. org/10.1097/INF.0000000000001823

23. Cadieux G, Redditt V, Graziano D, et al. Risk factors for varicella susceptibility among refugees to Toronto, Canada. J Immigr Minor Heal. 2017 Feb;19(1):6-14. https://doi.org/10.1007/s10903-015-0313-y

24. Grasso D, Boonsiri J, Lipschitz D, et al. Posttraumatic stress disorder: the missed diagnosis. Child Welfare. 2009;88(4):157-76.

25. Jones LK, Cureton JL. Trauma redefined in the DSM-5: rationale and implications for counseling practice. The Professional Counselor. 2014 Jul;4(3):257. https://doi.org/10.15241/lkj.4.3.257

26. Gadeberg AK, Montgomery E, Frederiksen HW, et al. Assessing trauma and mental health in refugee children and youth: a systematic review of validated screening and measurement tools. Eur J Public Health. 2017 Jun;27(3):439-46. https://doi.org/10.1093/eurpub/ckx034

27. Yurtsever A, Konuk E, Akyüz T, et al. An eye movement desensitization and reprocessing (EMDR) group intervention for Syrian refugees with post-traumatic stress symptoms: results of a randomized controlled trial. Front Psychol. 2018 Jun;9:493. https://doi.org/10.3389/ fpsyg.2018.00493

28. Edge S, Newbold B. Discrimination and the health of immigrants and refugees: exploring Canada's evidence base and directions for future research in newcomer receiving countries. J Immigr Minor Healt. 2013 Feb;15(1):141-8. https://doi.org/10.1007/s10903-012-9640-4

29. Pitman RK, Rasmusson AM, Koenen KC, et al. Biological studies of post-traumatic stress disorder. Nat Rev Neurosci. 2012 Nov;13(11):76987. https://doi.org/10.1038/nrn3339

30. Lehrner A, Yehuda R. Biomarkers of PTSD: military applications and considerations. Eur J Psychotraumatol. 2014 Aug;5. https://doi. org/10.3402/ejpt.v5.23797 